\title{
Spectral method of photoplethysmogram processing for screening of atherosclerosis
}

\author{
Anastasiya N. Markina*, and Aleksandr A. Fedotov \\ Samara National Research University, 34 Moskovskoye Shosse, Samara 443086, Russia \\ * e-mail: bel3289@yandex.ru
}

\begin{abstract}
The paper is devoted to the analysis of the existing indicators for the diagnostic of arterial vessels condition and the development of new ones based on the contour analysis of the pulse wave. The performed analysis of the techniques used to process the pulse wave signal has shown that the classical approach to the morphological analysis of pulse wave in terms of stiffness index and reflection index does not provide sufficient diagnostic accuracy due to the instable detection of the reflected wave maximum. A new technique of contour analysis based on spectral transformation of a sequence of replicated single fragments of the pulse wave is developed. The comparative analysis of the contour analysis methods has shown that the proposed method detects the changes of the pulse wave caused by diseases in the form of increased difference between the indicator values. (C) 2017 Journal of Biomedical Photonics \& Engineering.
\end{abstract}

Keywords: Pulse wave contour; Reflection index; Stiffness index; Form factor; Harmonic factor.

Paper \#3156 received 3 Feb 2017; revised manuscript received 24 Mar 2017; accepted for publication 29 Mar 2017; published online 7 Apr 2017. doi: 10.18287/JBPE17.03.020305. [Special Issue. Workshop "Biophotonics" of the XIII all-Russian Youth Samara conference-contest on optics and laser physics].

\section{References}

1. V. I. Kruglov, Cardiovascular Diseases, Leningrad publisher, Saint Petersburg (2009) [in Russian].

2. A. A. Fedotov, and S. A. Akulov, Measuring Transducers of Biomedical Signals in Clinical Monitoring Systems, Radio and Communications, Moscow (2013) [in Russian].

3. I. A. Mizeva, A. A. Dumler, and N. G. Muravyov, "Specific feature of pulse wave in patients with chronic arterial insufficiency of lower extremities," Russian Journal of Biomechanics 16(2), 83-94 (2012) [in Russian].

4. V. D. Koptev, T. I. Pospelova, and D. D. Tsyrendorzhiev, "Functional condition of vascular endothelium in patients with hemoblastosis before and after polychemotherapy," Siberian Oncology Journal 4(40), 20-24 (2010) [in Russian].

5. J. Allen, "Photoplethysmography and its application in clinical physiological measurement," Physiological measurement 28, R1-R39 (2007).

6. T. Weber, "Arterial Stiffness, Wave Reflections, and the Risk of Coronary Artery Disease," Circulation 109(2), 184-189 (2004).

7. C. Sandrine, "Contour analysis of the photoplethysmographic pulse measured at the finger," Journal of Hypertension 24(8), 1449-1456 (2006).

8. V. A. Berezovskii, and N. N. Kolotilov, Biophysical Characteristics of Human Tissues, Naukova dumka, Kiev, USSR (1990) [in Russian].

9. T. R. Dawber, H. E. Thomas, Jr, and P. M. McNamara, "Characteristics of the dicrotic notch of the arterial pulse wave in coronary heart disease," Angiology 24(4), 244-255 (1973).

10. M. Elgendi, "On the Analysis of Fingertip Photoplethysmogram Signals," Current cardiology reviews 8, 14-25 (2012).

11. R. M. Rangayyan, Analysis of biomedical signals. A practical approach, Fismatlit, Moscow (2007) [in Russian]. 
12. A. A. Fedotov, "Amplitude-time method of detecting the characteristic points of the pulse wave signal," Medical equipment 6, 22-28 (2012) [in Russian].

\section{Introduction}

Nowadays the pathologies of cardiovascular system occupy the leading position among all human diseases. The main cause of cardiovascular diseases (CVD) is the deformation of blood vessel walls, the atherosclerosis that arises due to the malfunction of lipid and protein metabolism and is accompanied by the deposition of cholesterol. The localisation of vascular pathology determines the consequences of the disease development, e.g., the atherosclerotic lesion of coronary arteries causes the development of ischemic heart disease (IHD). The atherosclerosis of brain vessels disturbs brain blood circulation, the possible consequences of which are the ischemic stroke and intracerebral bleeding [1].

Thus, the timely diagnostics of atherosclerosis development risk could make it possible to avoid multiple complications and fatal cases. Among the existing methods of clinical diagnostics of atherosclerosis, one can mention the angiography, the ultrasonic dopplerography, the laboratory examinations, and the analysis of pulse waves (PW), recorded using the sphygmographic or plethysmographic sensors [2]. The angiography is an invasive method of vascular examination that requires special preparation of the patient and the absence of contraindication. Hence, this diagnostic method is inapplicable to screening. The ultrasonic dopplerography allows the estimation of blood flow velocity and the visualisation of the vessel structure [3]. The laboratory examination of the vascular condition consists in determination of the level of endothelial dysfunction markers in blood [4].

The method most suitable for screening of atherosclerosis is the analysis of pulse wave signal, which is a noninvasive, relatively cheap and safe test, free of the patient's discomfort.

The aim of the present paper is to develop new approaches to the formation of diagnostic indicators for the assessment of arterial vessels condition.

\section{Morphology of pulse wave}

The pulse wave contour results from overlapping two waves. The direct one (the systolic wave $A_{1}$ ) is formed by the systolic blood volume transferred directly from the left ventricle to the arterial vessels of upper extremities during the systole. The reflected one (the diastolic wave $A_{2}$ ) is due to the reflection of the blood volume, transferred to the lower extremities via the aorta and the main arteries and directed back into the ascending aorta and then to the arterial vessels of upper extremities $[2,5,6]$.

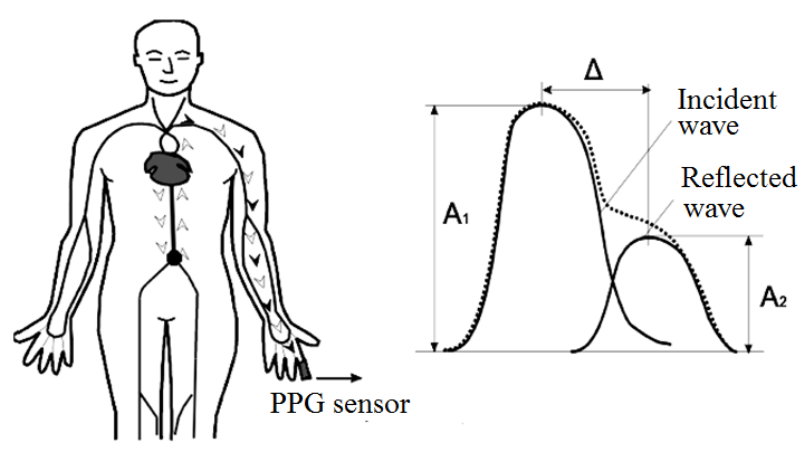

Fig. 1 Process of pulse wave formation.

The process of PW contour formation is illustrated in Fig. 1, where $>$ represents the direct pulse pressure wave, $\nabla$ shows the reflected pulse pressure wave, $\bullet$ is the aorta femoral bifurcation point, $A_{1}$ is the amplitude of the direct pulse wave, $A_{2}$ is the amplitude of the reflected pulse wave, $\Delta$ is the duration of pulse wave reflection.

The duration $\Delta$ of pulse wave reflection is determined by the propagation velocity given by the Moens-Korteweg equation:

$$
v=\sqrt{\frac{E h}{\rho d}},
$$

where $\quad v$ is the propagation velocity of the pulse pressure wave, $E$ is the Young modulus of the arterial wall, indicating its elasticity, $h$ is the thickness of the arterial wall, $\rho$ is the blood density, and $d$ is the artery diameter.

The parameters $h, \rho$, and $d$ are nearly similar for typical of blood vessels, so that the PW propagation velocity is mainly determined by the vessel elasticity [7]. Table 1 based on the data of Ref. [8] shows that with the decrease of vessel elasticity (increase of the Young modulus) the delay of the reflected pulse wave decreases. The presented values of the Young modulus were calculated basing on the results of ultrasonic scanning of common carotid arteries and common femoral arteries [8].

Thus, with increasing arterial stiffness, the reflected wave comes back faster, and its contribution is transferred from the diastolic component of the PW to the systolic one. The increased rigidity of arteries can appear not only with age, but also at earlier stages of life due to kidney diseases or diabetes mellitus. Figure 2 presents the classification of PW contours according to Dawber [9]. Class 1 is characterised by the expressed dicrotic notch, clear distinction between the direct and reflected wave, and different slope angles. Class 2 is characterised by the absence of notch, horizontal 
Table 1 Wave delay time $(\Delta)$ depending on the Young modulus $(E)$.

\begin{tabular}{|c|c|c|c|c|c|c|}
\hline & \multicolumn{3}{|c|}{$\begin{array}{l}\text { Carotid artery } \\
\text { (length } 8 \mathrm{~mm} \text {, diameter } 6.3 \mathrm{~mm} \text {, thickness } 1.75 \\
\mathrm{~mm})\end{array}$} & \multicolumn{3}{|c|}{$\begin{array}{l}\text { Femoral artery } \\
\text { (length } 56.6 \mathrm{~mm} \text {, diameter } 8 \mathrm{~mm} \text {, thicknes } \\
1 \mathrm{~mm} \text { ) }\end{array}$} \\
\hline & healthy & control & sick & healthy & control & sick \\
\hline$E, k P a$ & 522.15 & 825.44 & 1072.43 & 620 & 975.94 & 1122.76 \\
\hline$\Delta, m s$ & 21.38 & 17.01 & 14.92 & 206.94 & 164.94 & 153.78 \\
\hline $\begin{array}{l}\text { Blood density, } \\
\mathrm{kg} / \mathrm{m}^{3}\end{array}$ & 1036 & & & & & \\
\hline
\end{tabular}

separation of direct and reflected wave, and different slope angles. In Class 3 the notch and the change of slope angle are absent.

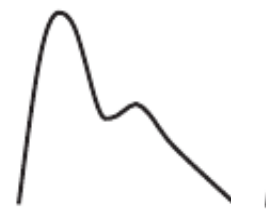

Class 1

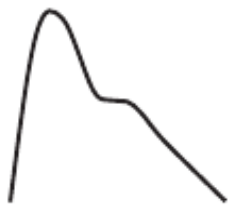

Class 2

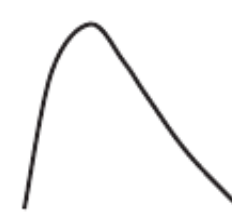

Class 3
Fig. 2 Modification of the pulse wave shape with age and in the presence of vascular diseases.

\subsection{Methods of contour analysis}

To date the processing of pulse wave signal is performed using the following amplitude-time parameters of the $\mathrm{PW}$ characteristic points:

- the reflection index $(R I)$ that characterises the reflection wave evidence and its contribution to the increase of the pulse arterial pressure

- $\quad R I=\frac{A_{2}}{A_{1}} \cdot 100 \%$;

- the stiffness index ( $S I$ ) determined by the velocity of pulse wave propagation

$S I=\frac{h}{\Delta}$,

where $h$ is the height of the patient

The methods of single or double differentiation of the signal are also in use (see Fig. 3) [10].

The first derivative allows the determination of the diastolic peak position (the point where the first derivative is zero), the reflection time (the time $\Delta$ between the systolic and the diastolic peaks, i.e., the points where the derivative changes its sign), and the PW rise time (the systolic push, the time during which the PW signal ascends from foot to peak).

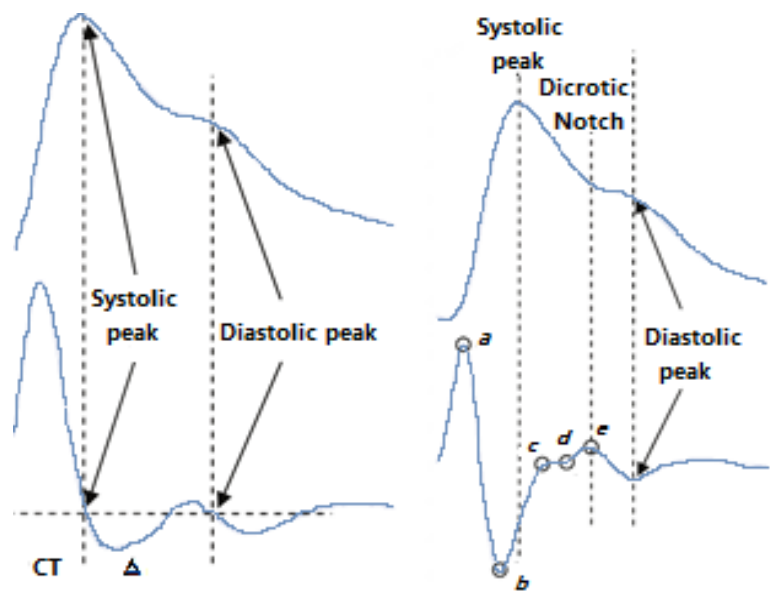

Fig. 3 The first and the second derivative of PW.

The second derivative of the pulse signal indicates the acceleration of the blood pulsation in the vessel. The contour of the second derivative of the pulse wave is formed by five sequential waves $a, b, c, d$, and $e$ that differ from each other in shape and amplitude. The relation of amplitudes of these waves is given by the coefficients that describe the vascular system condition $[7,10]$. The diagnostic indices determined basing on the analysis of the first and the second derivative are presented below:

- The coefficient $(b / a)$.

- The form factors $(F F)$ [11]

$$
F F=\frac{\sigma_{x^{\prime \prime}} / \sigma_{x^{\prime}}}{\sigma_{x^{\prime}} / \sigma_{x}}
$$

where

$\sigma_{x^{\prime \prime}}$ is the standard deviation of the second derivative of the considered fragment of the pulse wave signal from the mean value of the second derivative;

$\sigma_{x^{\prime}}$ is the standard deviation of the first derivative of the considered fragment of the pulse wave signal from the mean value of the first derivative;

$\sigma_{x}$ is the standard deviation of the considered fragment of the pulse wave signal from the mean value of the signal.

To study the existing methods of contour analysis by means of the certified computer photoplethysmograph ELDAR (ZAO "Novye Pribory", Russia), we formed an 


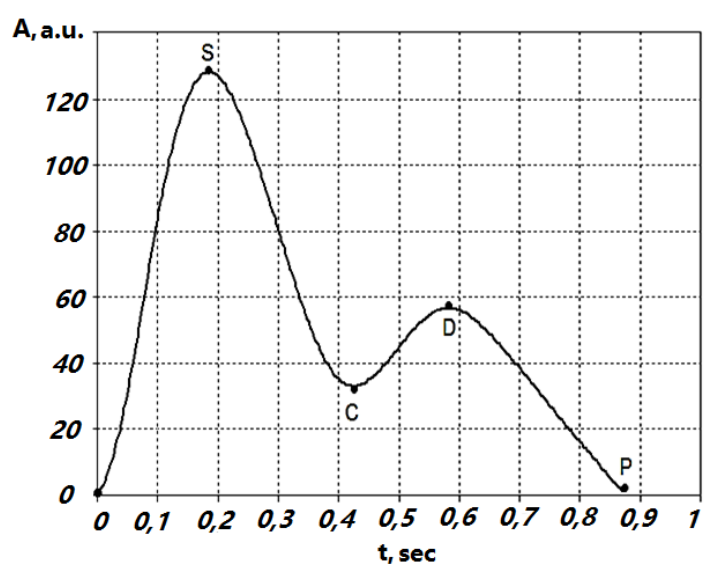

a)

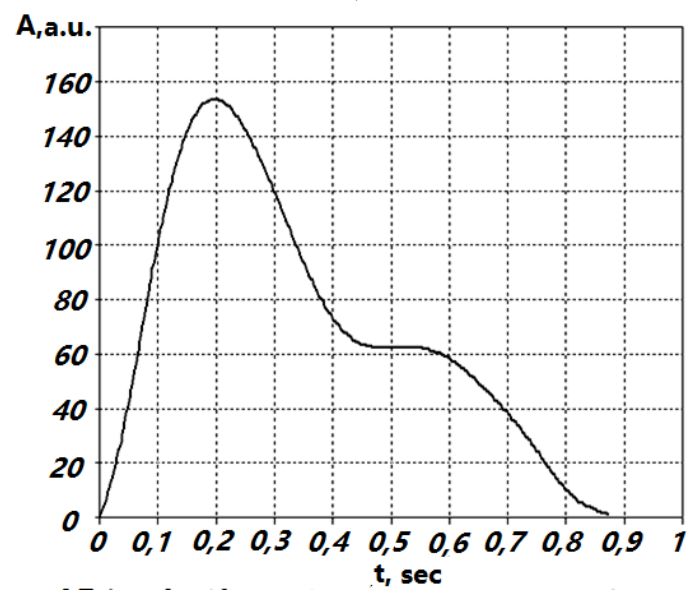

c)

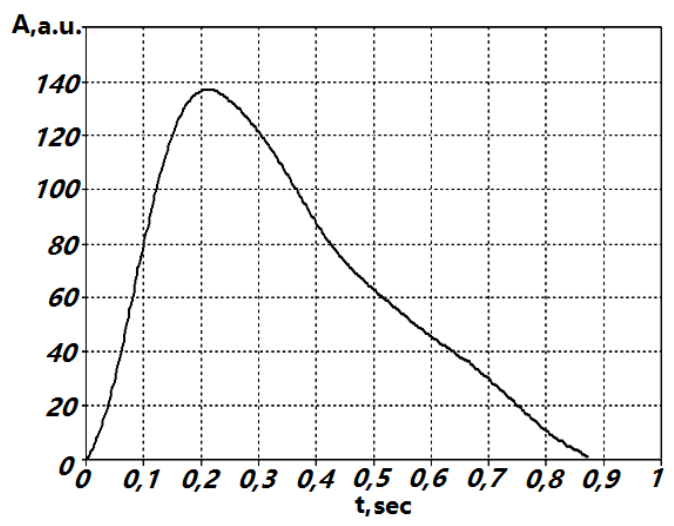

e)

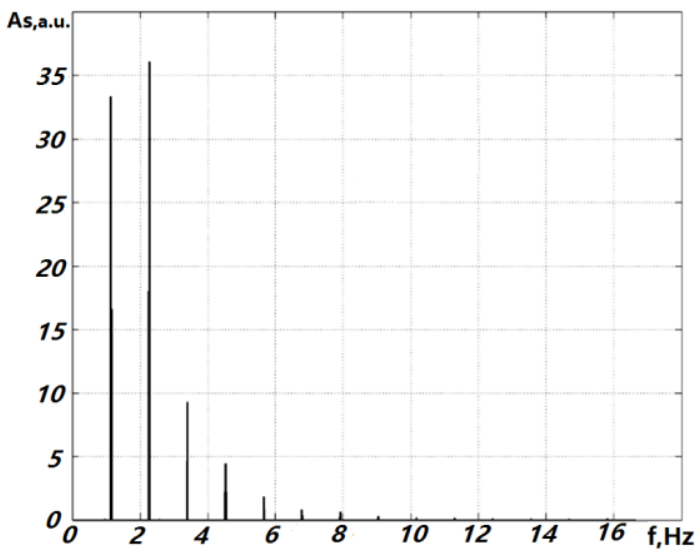

b)

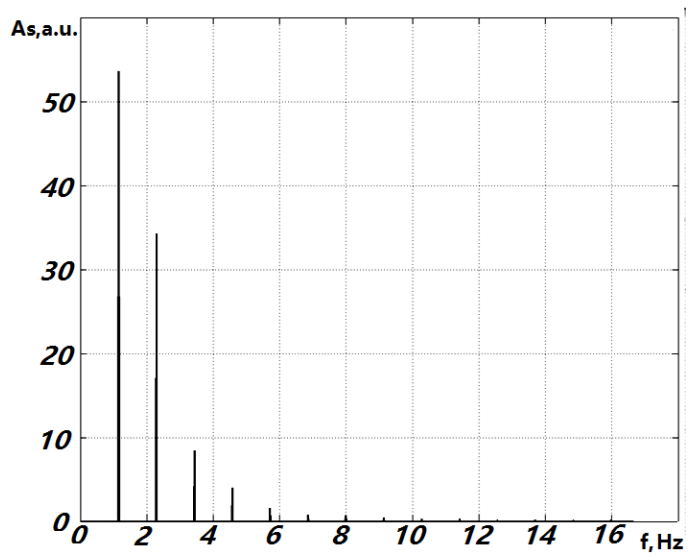

d)

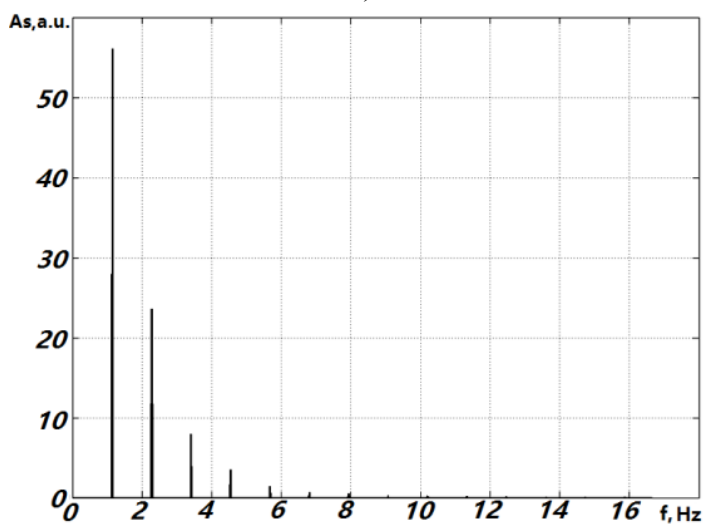

f)

Fig. 4 Typical fragments of pulse wave signal (a, c, e) and the amplitude spectrum of replicated fragments (b, d, f).

experimental array of pulse wave signals, recorded in groups of volunteers without diseases of vascular system, with age-related changes of vascular wall, and with cardiogenic pathologies. All patients confirmed their voluntary consent for being included in the examination. The preliminary analysis of the obtained signals revealed the presence of the first three types of the pulse waves.

\subsection{Results}

The transition from time domain to frequency domain by means of the spectral Fourier transform allows clearer assessment of the PW shape. To get the spectral assessment of the PW contour morphology, we applied the fast Fourier transform operations to the signal, obtained by sequential replication of single fragments of PW with the steady component removed. To form a fragment of the pulse wave, we used the amplitude-time detector of reference points, characterised by small detection error under the conditions of noise and 
Table 2 Diagnostic indices.

\begin{tabular}{cccccc}
\hline PW type & $\begin{array}{c}\text { Reflection } \\
\text { index, } \\
\%\end{array}$ & $\begin{array}{c}\text { Stiffness } \\
\text { index, } \mathbf{m} / \mathbf{s}\end{array}$ & $\begin{array}{c}\text { Form } \\
\text { factor }\end{array}$ & b/a & $\begin{array}{c}\text { Harmonic } \\
\text { factor }\end{array}$ \\
\hline Norm & $54 \pm 13$ & $7.5 \pm 0.73$ & $1.91 \pm 0.19$ & $-0.79 \pm 0.24$ & $0.93 \pm 0.17$ \\
\hline Risk & $61 \pm 15$ & $9.8 \pm 1.21$ & $1.48 \pm 0.16$ & $-0.80 \pm 0.18$ & $1.58 \pm 0.22$ \\
\hline Pathology & Impossible to determine & $1.21 \pm 0.12$ & $-0.81 \pm 0.26$ & $2.45 \pm 0.2$ \\
\hline
\end{tabular}

interference having different intensity and origin [12]. In the present amplitude-time detector, the characteristic points used to form a singular fragment were the points of maxima for the first derivative of the original signal. In this way, we avoid the detection of dicrotic maximum.

The analysis of the obtained spectral characteristics of the signal, formed by a sequence of different typical contours, has shown the presence of certain differences in the structure of amplitude spectrum (Fig. 4).

As an appropriate index to assess these differences, we proposed the harmonic factor, defined as the ratio of the first harmonic amplitude $A s_{1}$ and the second harmonic amplitude $A s_{2}$ in the spectrum:

$$
K S=\frac{A s_{1}}{A s_{2}} .
$$

The block diagram of the algorithm for complex processing of a photoplethysmogram (PPG) implemented in the Matlab software package is presented in Fig. 5.

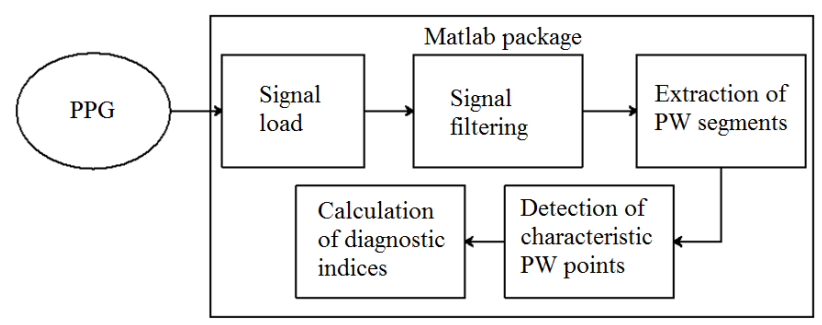

Fig. 5 Structure of the algorithm for photoplethysmogram processing.

\section{Conclusion}

The calculated diagnostic indices aimed to assess the arterial vessels condition basing on the PW contour analysis are presented in Table 2 .

With age, when the stiffness of blood vessels increases, or in the presence of pathology, the reflected wave propagates faster and stronger overlaps with the systolic component of PW. The bimodal shape of the signal transforms into a unimodal one (see Fig. 2, Class
3). Such reshaping makes the detection of the dicrotic maximum hardly possible.

The stiffness index and the reflection index do not allow one to obtain reliable diagnostic information in the group of volunteers with the pulse wave shape of the third type (Fig. 2). The coefficient $(b / a)$ characterises only the systolic part of PW. The harmonic factor based on the spectral analysis characterises the diastolic component better and allows the assessment of the signal shape change. The first two harmonics used to calculate the harmonic factor are less sensitive to noise. Thus, we get a more precise diagnostic parameter, in comparison with the form factor that takes the entire signal into account, as well as the entire spectrum of the signal. Moreover, the harmonic factor demonstrates the largest difference between three types of waves, compared to other indices.

The spectral analysis of the sequence of replicated PW fragments allows the assessment of the arterial vessels condition basing on the contour analysis. The main advantage of this method is that it is not necessary to detect precisely the amplitude-time parameters of the PW dicrotic notch. However, it is important to extract the pulse wave fragment correctly. 